\title{
ENERGY STORAGE BY PASSIVE ELASTIC STRUCTURES IN THE MANTLE OF SEPIA OFFICINALIS
}

\author{
N. A. CURTIN ${ }^{1, *}$, R. C. WOLEDGE ${ }^{2}$ AND Q. BONE 3 \\ ${ }^{1}$ Cellular and Integrative Biology, Division of Biomedical Sciences, Fleming Building, Imperial College School of \\ Medicine, London SW7 2AZ, UK, ${ }^{2}$ UCL Institute of Human Performance, Royal National Orthopaedic Hospital \\ Trust, Brockley Hill, Stanmore, Middlesex HA7 4LP, UK and ${ }^{3}$ Marine Biological Association, The Laboratory, \\ Citadel Hill, Plymouth PL1 2PB, UK \\ *e-mail: n.curtin@ic.ac.uk
}

Accepted 14 December 1999; published on WWW 10 February 2000

\begin{abstract}
Summary
The passive elastic properties of the mantle of the cuttlefish Sepia officinalis have been characterized in experiments on intact mantle and on pieces cut from the mantle. The mantle was found to be very compliant over a wide range of circumferential strains, corresponding to a change in mantle circumferential strain of 0.45 . Beyond this range of strain, the mantle was much stiffer, in both the circumferential direction, $0.542 \pm 0.025 \mathrm{MPa}$ (mean \pm

this elastic work could contribute to refilling the mantle after a jet. Our estimates of the work done during a cycle of jetting and refilling show that such elastic work is small (approximately 1\%) compared with the contractile work done by the circular muscle fibres. However, although the elastic work is almost negligible in the overall energy budget, it is probably sufficient to power refilling of the mantle.
\end{abstract} S.E.M., $N=51$ ) and through the thickness of the mantle wall, $0.152 \pm 0.041 \mathrm{MPa}(N=11)$. Almost $80 \%$ of the work done on the tissue during compression in the circumferential direction was recovered during elastic recoil of the tissue;

Key words: cuttlefish, Sepia officinalis, elastic energy storage, cephalopod, locomotion, mantle.

\section{Introduction}

Cephalopod mantles are used for jet propulsion and respiration. The muscle fibres that generate pressure within the mantle cavity to produce the jet and respiratory flow are arranged with their long axes oriented around the circumference of the mantle (Fig. 1). These muscle fibres are referred to as circular muscle fibres. The mantle cavity must refill after outward flow has finished. It has been suggested that elastic recoil (shortening) of collagen strands that span the thickness of the mantle causes refilling. These strands are stretched as the circular muscle fibres shorten and thicken the mantle wall during jetting or respiratory exhalation. Gosline and Shadwick (1983a,b) have measured the passive elastic properties of the mantle of the squid Loligo opalescens, and calculated that the energy that can be stored in this way represented a significant fraction of the work done by the circular muscle fibres during active shortening. However, because they did not measure the properties of the active muscle, this suggestion could not be tested quantitatively. Active muscle properties have been determined recently for the circular muscle fibres in another cephalopod, the cuttlefish Sepia officinalis (Milligan et al., 1997). We have observed the passive, elastic properties of the mantle in this species and have made a direct comparison of circular muscle fibre work with elastic energy storage.
Fig. 1 shows diagrammatically the main structures within the mantle. The bulk of the tissue is composed of the circular muscle fibres that run around the circumference of the mantle. Smaller bundles of muscle fibres run radially through the thickness of the wall. These radial muscle fibres contract and thin the mantle wall during hyperinflation before a jet (Gosline et al., 1983) and during the inspiration phase of Sepia officinalis respiration (Bone et al., 1994). Both surfaces of the mantle are covered by a tunic, which contains large collagen strands arranged at specific angles (Ward and Wainwright, 1972). Kinematic studies show that the mantle does not elongate during jetting (Ward, 1972; Packard and Trueman, 1974), and it seems likely that the collagen strands in the tunic prevent elongation. Connective tissue strands are also present within the muscular part of the mantle wall, where they are arranged in three planes (Bone et al., 1981). These strands run at a more variable angle than those in the tunics. One set of strands is found running in the $C L$ plane (as defined in Fig. 1), and the other two sets run obliquely through the thickness of the mantle in the $C T$ plane and in the $L T$ plane (as defined in Fig. 1).

Most of these connective tissue strands will be stretched when the circular muscle fibres shorten and the mantle gets thicker. If these strands were to act as independent springs 
Fig. 1. Diagram of a piece cut from the mantle of Sepia officinalis showing two sets of connective tissue strands and the radial and circular muscle fibres. $C$ represents the circumferential dimension, $T$ is the thickness of the mantle and $L$ is the longitudinal dimension of the piece of mantle tissue. The tunics, which are connective tissue layers covering the inner and outer surfaces of the mantle, are not shown.

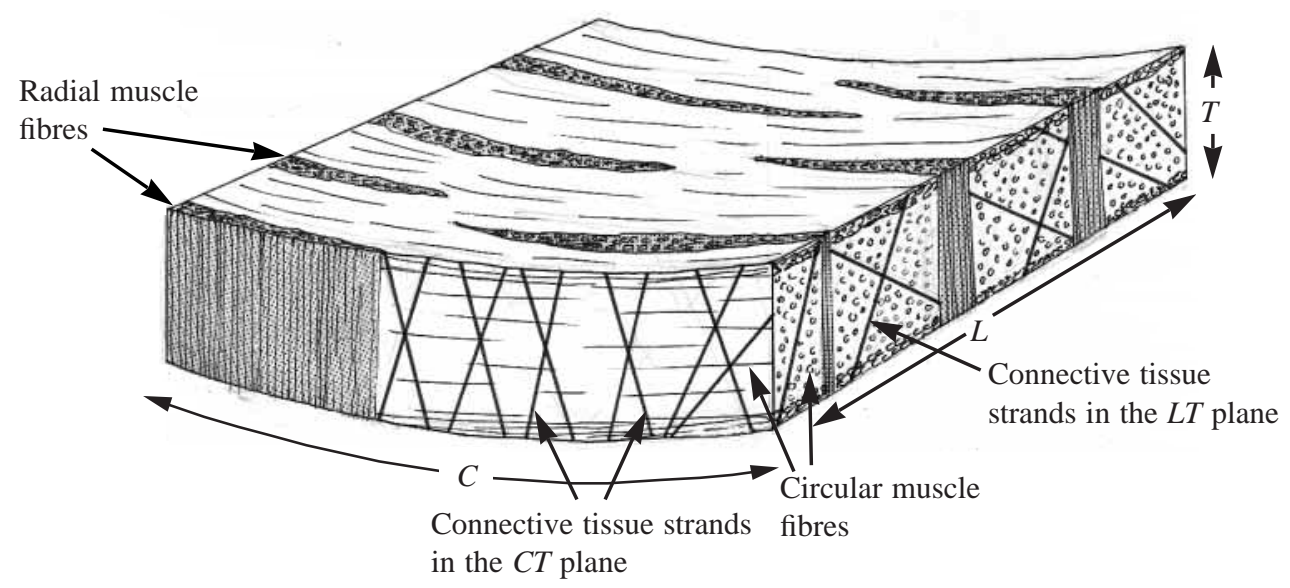

connected to the tissue only at the inner and outer surfaces, then the elastic properties of a piece cut from the mantle would depend on the dimensions of the piece. This is because some of the strands are cut and thus rendered ineffective. The proportion that are cut and so eliminated will be greater the smaller the piece of mantle. Thus, if this simple independent spring model is correct, the stiffness of large pieces will be greater than that of small pieces of mantle. To investigate whether this is so, we have measured the elastic properties of pieces with a range of $C$ values and a range of $L$ values.

\section{Materials and methods}

Experiments were performed on specimens of Sepia officinalis. The animals were anaesthetized in $5 \%$ ethanol in sea water and then decapitated.

\section{Compression of pieces of mantle}

The mantle was cut along the midline of the ventral surface and opened out. After removal of the internal organs, the mantle was kept in ice-cold sea water, and the pieces required for the experiments were removed from either side of the initial cut, by cutting through the complete thickness of the mantle with a scalpel blade. The approximate size ranges were $C=5.5-13 \mathrm{~mm}$ and $L=7-12 \mathrm{~mm}$. The value of $T$ (mantle thickness) was approximately $7 \mathrm{~mm}$. During the experiment, the mantle pieces were kept in artificial sea water and maintained at approximately $14{ }^{\circ} \mathrm{C}$. All experiments were completed within $3 \mathrm{~h}$ of killing the animal. The pieces were immersed in sea water after the mechanical measurements had been made. Later on the same day, each piece was blotted with filter paper to remove excess sea water and weighed.

\section{Apparatus and test protocol}

Fig. 2 is a plan view diagram of the apparatus used to measure the passive mechanical properties. The piece of mantle being tested was placed in a Petri dish between a steel bar, on which strain gauges were mounted, and a Perspex block which could be advanced towards the strain gauge bar by a manually operated micrometer drive. A linear potentiometer was held by a spring in contact with the micrometer-driven

block. The outputs from the linear potentiometer and the strain gauge bridge circuit were recorded using a DAS-1601 data acquisition board (Keithley Data Acquisition) in a PC under the control of specially written software using ViewDac (Keithley Data Acquisition). After inserting the mantle piece between the restraints, the micrometer was advanced until the output of the strain gauges changed by $100 \mathrm{mV}$ (equivalent to approximately $40 \mathrm{mN}$ ). At this point, the reading of the micrometer was noted, and from this reading the starting dimension, $D_{\mathrm{s}}$, was found. The tissue was then compressed by advancing the micrometer in approximately 12 steps of $0.1-$ $0.3 \mathrm{~mm}$; the compression was then released in steps of the same size. Force and displacement were recorded simultaneously at $50 \mathrm{~ms}$ intervals during the period of approximately $40 \mathrm{~s}$ covering these movements. Each mantle piece was subjected

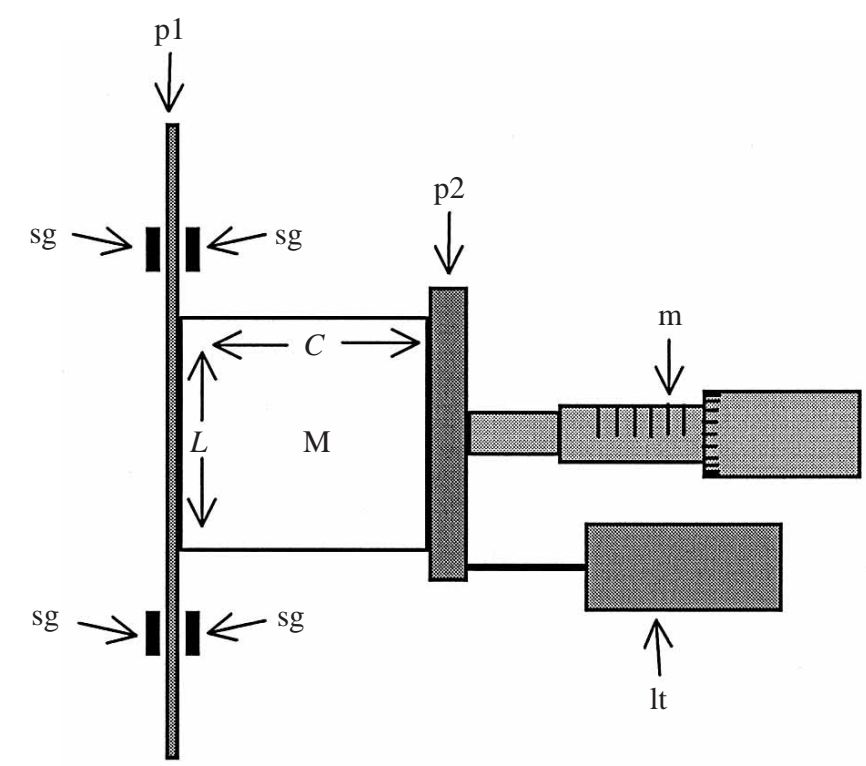

Fig. 2. Diagram of the apparatus (plan view, not to scale) and mantle piece arranged to be compressed in the circumferential direction. M is the piece of mantle, $C$ and $L$ are the dimensions shown in Fig. 1. $\mathrm{p} 1$ is a fixed plate with strain gauges (sg), and p2 is a movable plate, the position of which is controlled by the micrometer (m) and detected by the length transducer (lt). See text for further details. 
to two cycles of compression and release. Some displacements were made more rapidly, approximately four times faster.

In experiments on pieces of mantle from two Sepia officinalis, the compression was in the circumferential direction, distorting the tissue as shortening of the circular fibres does. One further animal was used for experiments in which the thickness $(T)$ was compressed, distorting the tissue as shortening of the radial fibres does. The length $(L)$ of each piece was measured before compression. The starting dimension of the circumference $(C)$ or of $T$ for each piece was found from the initial micrometer reading. The third dimension ( $T$ or $C$ ) was found by dividing the blotted wet mass by the other two dimensions; we have assumed the density of the tissue to be $1060 \mathrm{~kg} \mathrm{~m}^{-3}$ (Mendez and Keys, 1960).

\section{Pressure/volume relationship in intact mantle}

Experiments of this type were performed on two specimens of Sepia officinalis. The mantle opening was tied securely to a tapered rubber stopper that fitted closely into the opening. The seal between the mantle and stopper was tested and judged to be adequate if a stable pressure gradient could be maintained for several minutes across the mantle wall (see below). Measured volumes of sea water could be added to and removed from the mantle via a rigid tube through a hole in the stopper. The mantle and stopper were placed in a large glass cylinder containing sea water. The rigid tubing was marked with calibrated graduations so that changes in fluid level both inside and outside the tube could be measured. Pressure was calculated from the difference in the level of the sea water inside and outside the rigid tubing, using a value of $1025 \mathrm{~kg} \mathrm{~m}^{-3}$ for the density of sea water at $20^{\circ} \mathrm{C}$ (the temperature at which the experiments were carried out). The volume of sea water inside the mantle was measured for zero pressure difference across the mantle wall.

\section{Results}

Fig. 3 shows interference contrast micrographs of a section of fresh mantle tissue cut in the $C T$ and the $L T$ planes (the transverse direction is vertical in both micrographs). The oblique strands of connective tissue are clearly visible. The angle between the strand and the horizontal (corresponding to the mantle surface) was measured for approximately 30 such strands in the micrographs shown in Fig. 3. Fig. 4 shows the distribution of the angles of the connective tissue strands in the two planes. On average, the strands run at larger angles in the $C T$ plane $(P=0.001$, unpaired $t$-test $)$.

\section{Compression of pieces of mantle}

Fig. 5A shows an example recording of force produced during compression and subsequent release of a piece of mantle in the circumferential direction. During the first $1 \mathrm{~mm}$ of compression (approximately 10\% strain), there is little resistance; thereafter, the force rises more steeply. From these recordings, the stress/strain curve in Fig. 5B was plotted. Here, force is expressed as stress (force divided by the area, $T \times L$, see Fig. 1) and strain is
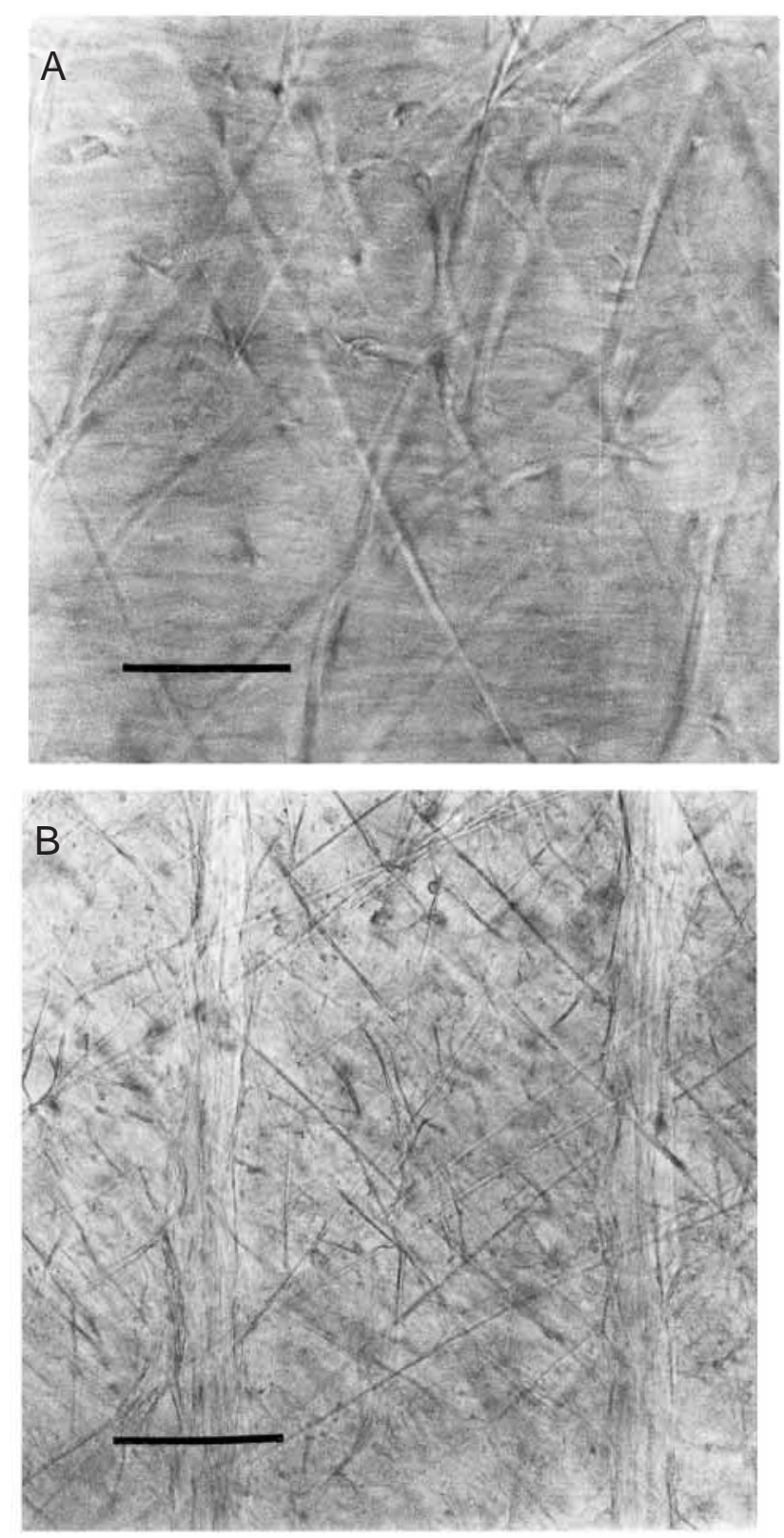

Fig. 3. Interference contrast micrographs of fresh, unstained sections of Sepia officinalis mantle. (A) View of the $C T$ plane illustrated in Fig. 1. The transverse direction is vertical. The fine horizontal lines are the long axes of the circular muscle fibres. The bright, oblique lines are the connective tissue strands. Scale bar, $50 \mu \mathrm{m}$. (B) View of the $L T$ plane illustrated in Fig. 1. The transverse direction is vertical. The vertical stripes are groups of radial muscle fibres. The dark, oblique lines are the connective tissue strands. Scale bar, $50 \mu \mathrm{m}$.

the ratio of change in $C$ to the initial value of $C$. The initial value of $C$ was the length at which compression first produced measurable force (approximately $40 \mathrm{mN}$ ). The stress increased gradually for the first 0.15 increase in strain, and then more steeply for the next 0.12 increase in strain. In this steeper part of the curve, stress is a linear function of strain, i.e. the stiffness is constant. A linear regression (shown in Fig. 5B) is fitted to this part of the curve; its slope (the stiffness) is $0.498 \mathrm{MPa}$ and the 

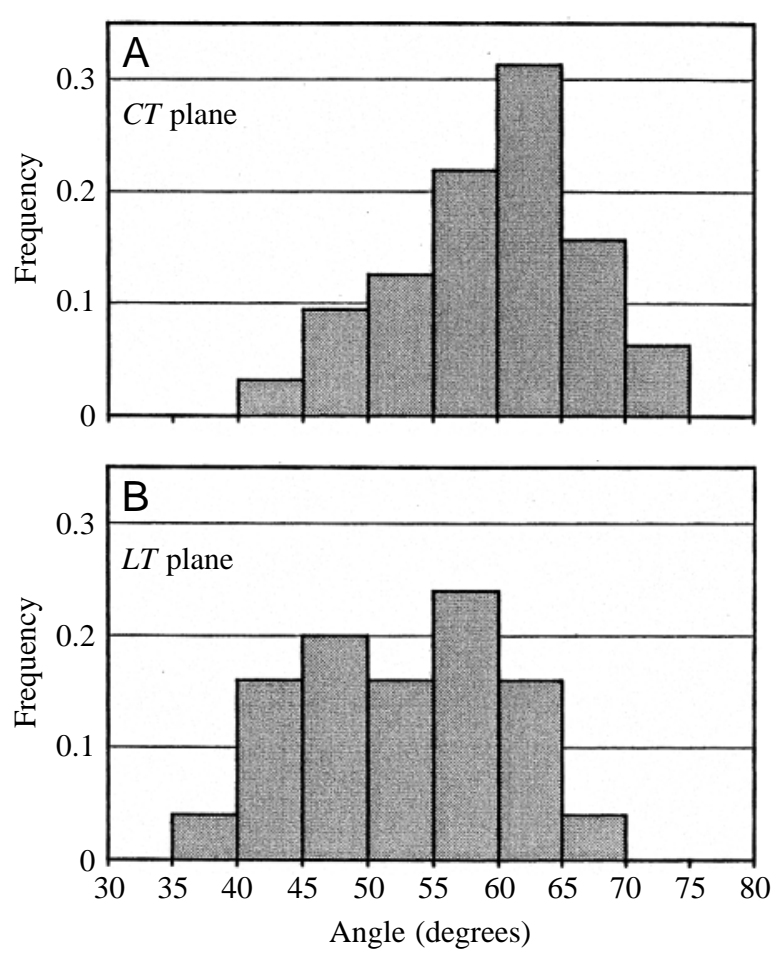

Fig. 4. (A,B) Histograms showing the distributions of angles of the connective tissue strands in the micrographs shown in Fig. 3A and Fig. 3B, respectively. The angles were measured between the horizontal, which corresponds to the surface of the mantle, and the strand. (A) Angles of 32 strands in the $C T$ plane. Mean value $59.75^{\circ}$. (B) Angles of 25 strands in the $L T$ plane. Mean value $52.85^{\circ}$. See also Fig. 1.

intercept on the strain axis is 0.164 . During release of strain, the stress was slightly less than during the increase in strain. In this example, the work done by the slice during release was $77.3 \%$

Table 1. Results of compression of cuttlefish mantle pieces in the circumferential and transverse directions

\begin{tabular}{lcccccc}
\hline & \multicolumn{2}{c}{$\begin{array}{c}\text { Circumferential } \\
\text { compression }\end{array}$} & & & \multicolumn{2}{c}{$\begin{array}{c}\text { Transverse } \\
\text { compression }\end{array}$} \\
\cline { 2 - 3 } \cline { 5 - 6 } & Mean & $N$ & & Mean & $N$ \\
\hline Stiffness $(\mathrm{MPa})$ & $0.542 \pm 0.025$ & 51 & & $0.152 \pm 0.041$ & 11 \\
Intercept $\left(D / D_{\mathrm{s}}\right)$ & $0.163 \pm 0.008$ & 51 & & $0.153 \pm 0.049$ & 11 \\
Resilience & $0.790 \pm 0.014$ & 51 & & $0.329 \pm 0.142$ & 11
\end{tabular}

Values are means \pm S.E.M.; $N$ is the number of measurements.

The 51 measurements during compression in the circumferential direction were performed on 27 pieces from two Sepia officinalis. The 11 measurements during compression in the transverse direction were performed on six pieces from one $S$. officinalis.

Stiffness is the slope of the linear part of the observed stress versus strain relationship (see Fig. 5B).

$D$ is the dimension of the piece of tissue and $D_{\mathrm{s}}$ is the starting value (see text) of dimension $C$ for compression in the circumferential direction and dimension $T$ for compression in the transverse direction (see Fig. 1).

Resilience is work done by the piece of tissue during elastic reextension/work done on the piece to compress it. of that done on the slice during compression (i.e. resilience was 0.773). Similar experiments were performed with 27 pieces of mantle from two Sepia officinalis.

In these experiments, the distortion of the mantle is similar to that produced by shortening of the circular muscle fibres. To examine also what happens when the radial muscle fibres shorten, we carried out further experiments in which mantle pieces were compressed in the transverse direction. These results were obtained from six pieces from one Sepia officinalis and were very similar to those for compression in the circumferential direction, except that the resistance to compression was less.

The results of observations of compression in the circumferential direction and compression in the transverse direction are summarized in Table 1, which shows the values
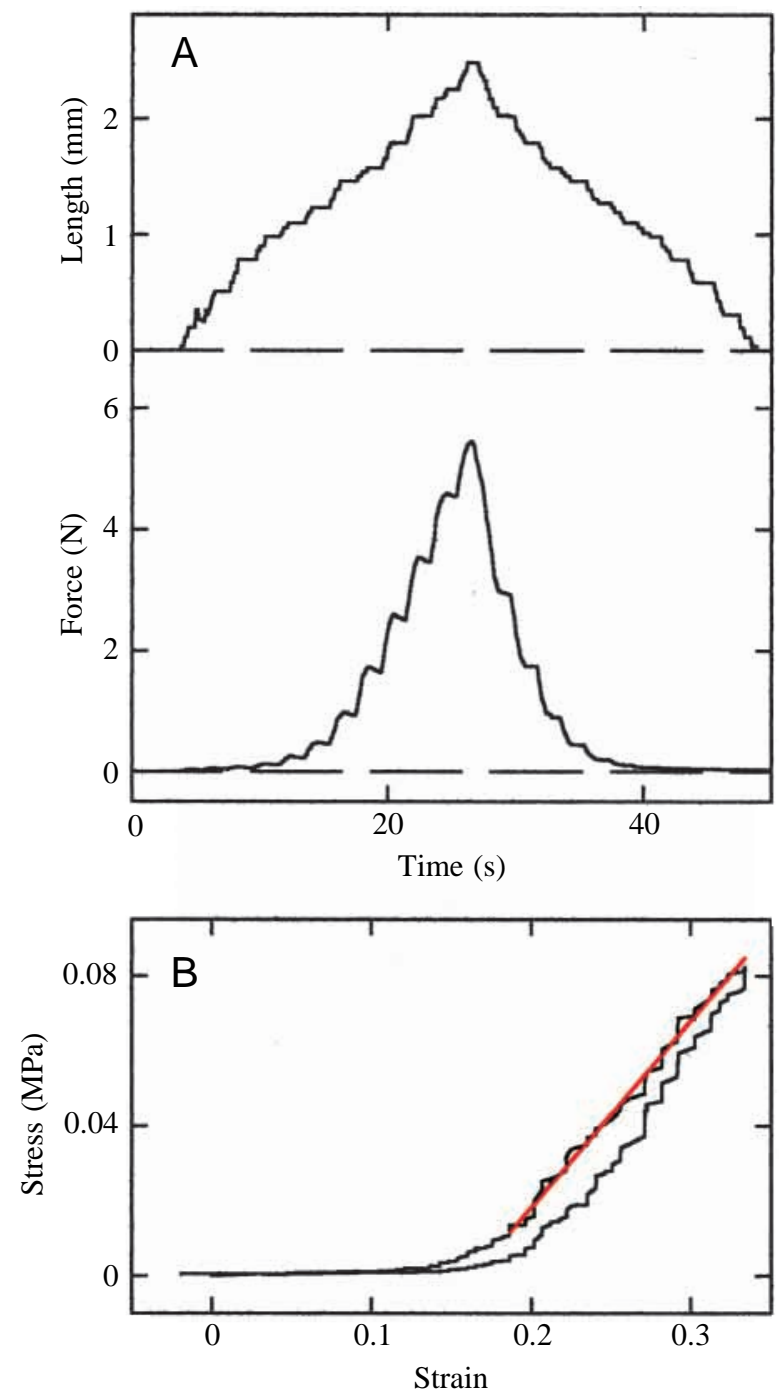

Fig. 5. (A) Example recordings of length change and force exerted by a piece of mantle during compression in the circumferential direction (see Fig. 1). (B) The results shown in A plotted as a stress/strain curve, where stress is force/area and strain is change in length/initial length. The regression line (red) was fitted to the data for strains greater than 0.19 and its slope was taken as the stiffness. See Table 1 for the summary. 
of stiffness in the linear region, the intercept of the linear regression on the strain axis, and the resilience. Tests made with a small number of the slices (six) from one Sepia officinalis suggest that the resilience is even higher when the movement is made more quickly (approximately four times faster, with compression and release complete in approximately $10 \mathrm{~s}$ compared with approximately $40 \mathrm{~s}$ ).

\section{Effects of size on stiffness}

As stated in the Introduction, it has been suggested that the stiffness of the mantle is due to the presence of connective tissue strands running obliquely through the thickness of the mantle. If this is the case, then the number of strands in a given piece would be proportional to the area, $L \times C$. This arrangement is shown diagrammatically in Fig. 6C.

The stiffness of mantle pieces cut with different $C$ values is compared in Fig. 6A. In these experiments, the range of $C$ values was $5.5-13 \mathrm{~mm}$ while keeping the other dimensions approximately constant at $L \approx 10 \mathrm{~mm}$ and $T \approx 7 \mathrm{~mm}$. The abscissa in this figure is the ratio $C / T$. The results show that the stiffness falls as $C$ increases. Fig. 6B shows results from similar experiments in which the value of $L$ varied among the pieces. The range of $L$ values was $7-12 \mathrm{~mm}$, with approximately constant values of $C(\approx 8 \mathrm{~mm})$ and $T(\approx 6 \mathrm{~mm})$. Again there is a tendency for the stiffness to be less when $L$ is greater. In plotting these figures, the stiffness has been calculated by expressing the stress as force relative to $C \times L$, the area to which the hypothetical strands attach.

In these experiments, the work was calculated by integrating

Fig. 6. (A,B) Symbols show the stiffness of mantle pieces of different sizes. Stiffness was measured during compression in the circumferential direction. Stiffness was taken as the slope of the linear part of the stress/strain curve (see Fig. 5B). Size is expressed as the ratio of the dimension that was varied (either $C$ or $L$, where $C$ is the circumferential dimension and $L$ is length; see Fig. 1) to $T$, the thickness of the mantle wall, which remained uncut. The vertical lines join results of repeated tests on the same piece of mantle. In A, size was varied by cutting out mantle pieces with different values of $C$. In B, size was varied by varying the dimension $L$. Stress is force/ $(C \times L)$, where $C \times L$ is the area to which the hypothetical springs attach and is thus an index of the number of springs. The solid curved lines show the predicted relationship for intact, connective tissue strands acting as independent, linear springs. The following equation was used: $S_{\text {predicted }}=\left[1-1 /\left(\tan \theta_{\mathrm{C}} C / T\right)\right]+\left[1-1 /\left(\tan \theta_{\mathrm{L}} L / T\right)\right]$, where the predicted stiffness ( $\left.S_{\text {predicted }}\right)$ rises to 2 for an infinitely large piece of mantle (see right-hand axis), $\theta_{\mathrm{C}}$ is the angle between the strands in the $C T$ plane and the circumferential axis, and $\theta_{\mathrm{L}}$ is the angle between the strands in the $L T$ plane and the longitudinal axis. $\theta_{\mathrm{C}}$ was taken to be $59.75^{\circ}$ and $\theta_{\mathrm{L}}$ to be $52.85^{\circ}$, the mean values from Fig. 4A,B. (C) Diagram of a mantle piece showing the $C, T$ and $L$ dimensions as in Fig. 1. The green diagonal lines in the $C T$ and the $L T$ planes indicate connective tissue strands. The dots on the $C L$ surface indicate the sites of attachment of the strands. The broken lines mark the cut that would be made to reduce $C$ (blue) or $L$ (red) for the experiments giving the results shown in A and $\mathrm{B}$ respectively. The horizontal arrows show the direction of compression force for these experiments. recordings of force versus length change. The resilience of the mantle pieces (the work done by the mantle during recoil/the work done on it during compression) was evaluated for pieces of different size. Fig. 7A,B shows how resilience varies with $C$ and $L$, respectively. In both cases, resilience decreased slightly as the size of the piece increased.

\section{Pressure/volume relationship in intact mantle}

Sea water was added or removed from intact whole mantle, and the pressure difference was found from the levels of sea water inside and outside the rigid tube that had been inserted into the mantle (see Materials and methods). Fig. 8 shows the pressure/volume relationship determined in this way for two Sepia officinalis mantles. The results show that the mantle was very compliant when the pressure gradient was small. Changes in fractional volume within the range +1.0 to -0.5 were produced by pressure changes of less than $0.2 \mathrm{kPa}$. Beyond this
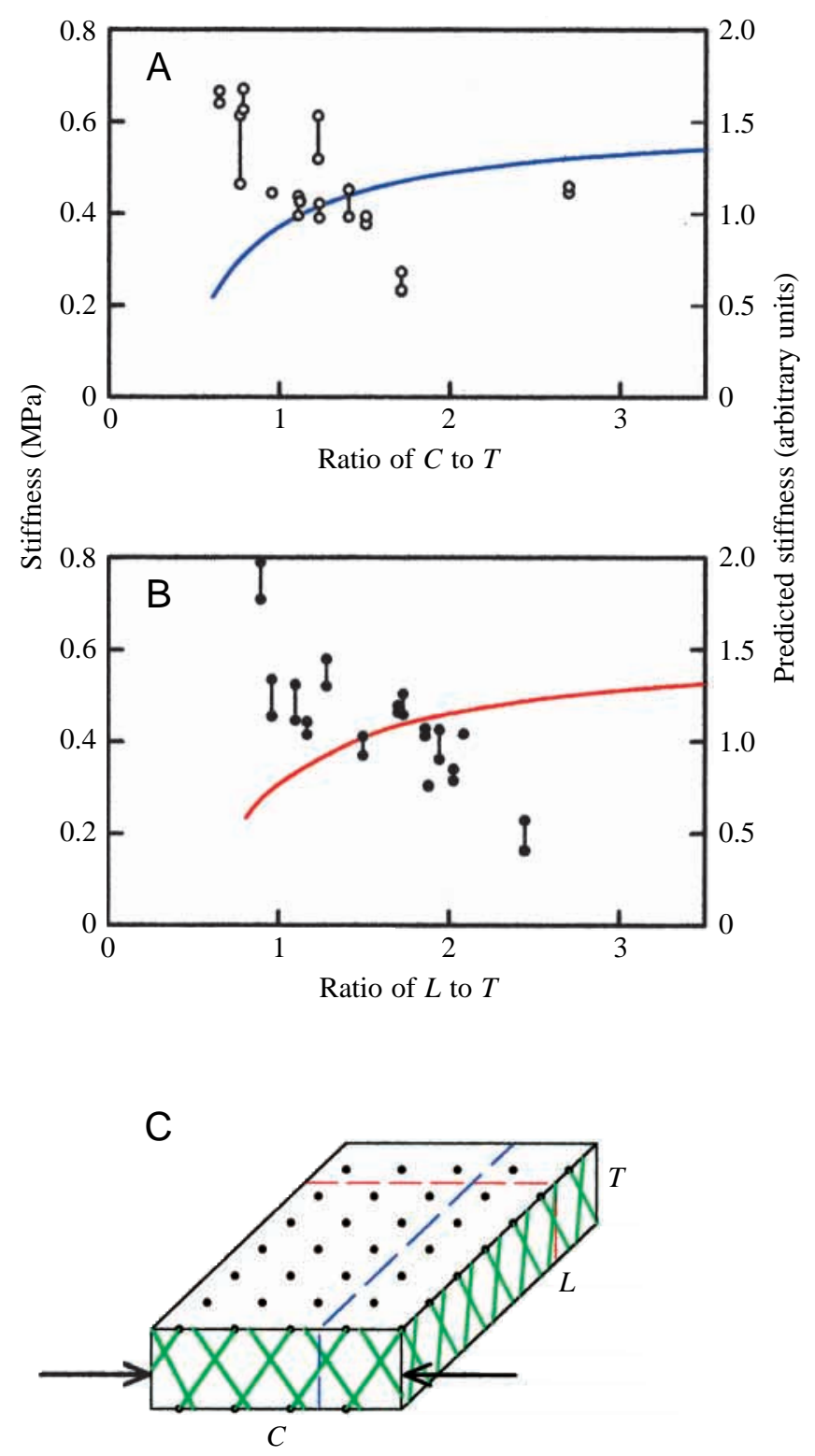

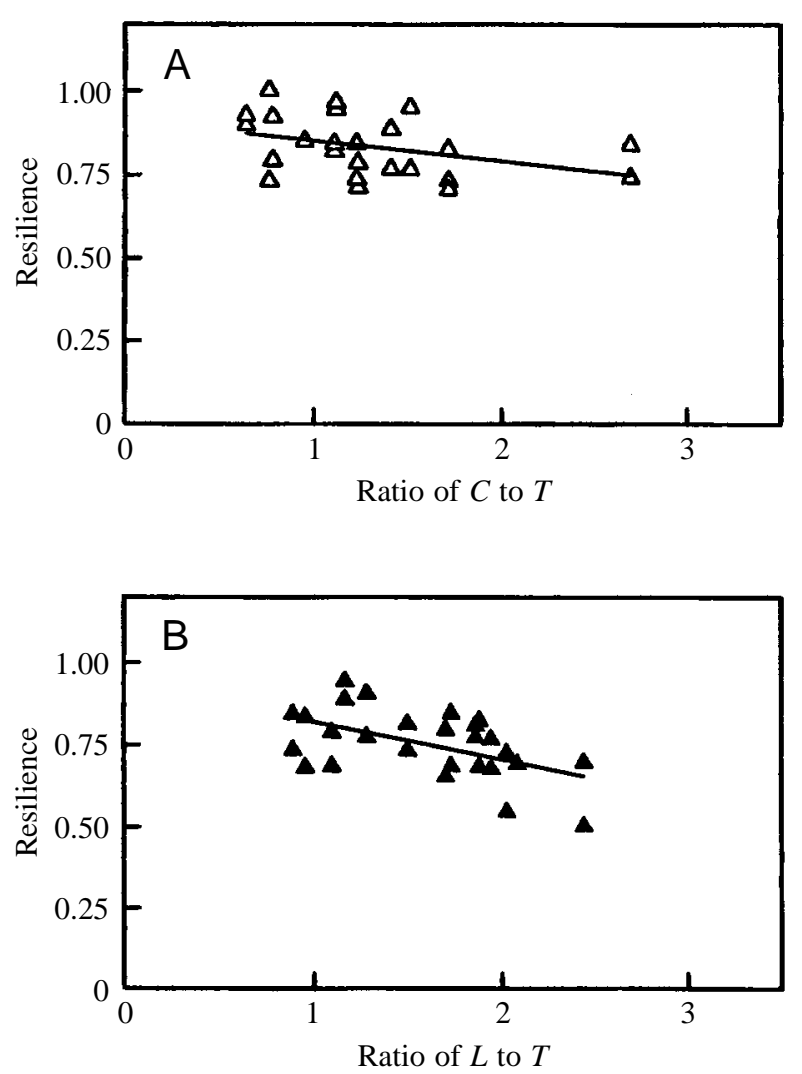

Fig. 7. (A,B) Symbols show values of resilience of mantle pieces of different sizes, measured during compression and release in the circumferential direction. Resilience is the work done by the mantle piece during release/work done on it during compression. Results are for pieces of different sizes. In A, size was varied by cutting out mantle pieces with different values of $C$ (see Fig. 1). In B, size was varied by varying the dimension $L . T$ is the thickness of the mantle wall. The regression lines are shown. For A, $y=-0.060 \pm 0.032+0.910 \pm 0.045 x \quad(P=0.075, \quad N=24)$. For $\mathrm{B}$, $y=-0.114 \pm 0.037 x+0.933 \pm 0.062(P=0.005, N=27)$. Values are means \pm S.E.M.

range, the mantle was stiffer, resisting volume change more strongly.

\section{Discussion \\ Stiffness values}

Our results for compressing pieces of Sepia officinalis mantle show that when it is made thicker by compressing in the circumferential direction there is elastic resistance to the distortion. The resilience of whatever structure provides this resistance is high. In these respects, our results resemble those of Gosline and Shadwick (1983a,b), who reported similar experiments made with pieces cut from the mantle of the squid Loligo opalescens. Oblique connective tissue strands running through the thickness of the mantle are present in both the cuttlefish Sepia officinalis and squid and run at similar angles. However, the stiffness that we measured, $0.542 \pm 0.025 \mathrm{MPa}$ (mean \pm S.E.M., $N=51$ ), is much less than the value of $2 \mathrm{MPa}$

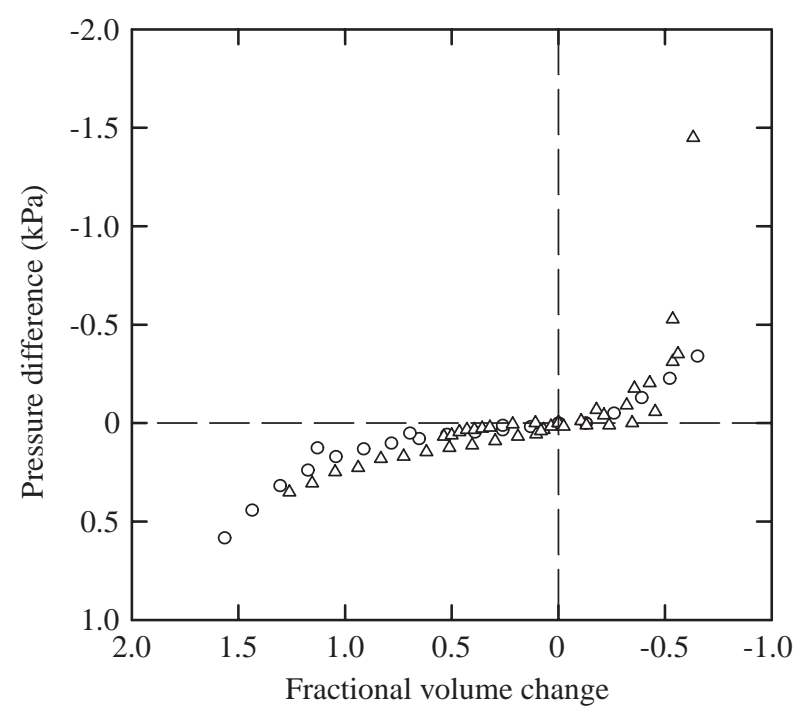

Fig. 8. The relationship between fractional volume change and the pressure difference between the inside and outside of intact, whole mantle. Note that the abscissa shows decreasing volume to the right. Results are from two Sepia officinalis $(\bigcirc, \triangle)$.

reported by Gosline and Shadwick (1983a,b). If the resistance is due to the oblique collagen strands, such a difference could, for example, arise if there were more or stiffer strands in the squid, which produces a more powerful jet than that of Sepia officinalis. There is at present no quantitative evidence as to the number, size or stiffness of these strands in either species.

\section{Relationship between stiffness and size}

If the resistance to compression of the mantle is due mainly to the action of the collagen strands running in the $C T$ and $L T$ planes, and if these strands act independently of each other, then the resistance of a piece cut from the mantle will be dependent on how many of these strands remain intact. In smaller pieces, a higher proportion of the strands will have been cut. Therefore, the resistance to compression, when normalised by the area $C \times L$, would tend to be less in smaller pieces. To illustrate this effect, we show in Fig. 6A,B predictions of how the stiffness would vary with the dimension of the piece if all the resistance came from fibres running at the mean angle that we have observed for each plane (see Fig. 4). In making this calculation, we have assumed that, in the intact mantle, half the total resistance to compression is due to strands in the $C T$ plane and half to those in the $L T$ plane.

The predicted stiffness rises as the ratio $C / T$ (or $L / T$ ) increases. This is not what was observed; the measured values of stiffness were actually smaller for the larger slices. The observations seem to be incompatible with the suggestion that the stiffness is due to these connective tissue strands acting as simple, independent, linear springs. If the strands are not independent, but are linked to each other or to the matrix to form a meshwork, the stiffness might be independent of the size of the piece. However, the observations seem to be incompatible with this suggestion also because stiffness 
actually decreases as $C / T$ (or $L / T$ ) increases. We can suggest three possible reasons for the discrepancy. (i) The damaged tissue adjacent to the cut surface is the major site of the resistance to distortion. Its effect on the total stiffness diminishes as the size of the piece increases and the proportion of undamaged tissue increases. (ii) In larger pieces, the structure resisting the compression can more easily accommodate distortion by, for example, bending rather than compressing. Macroscopic buckling of the mantle pieces did not occur in these experiments, but we could not have detected such processes on a microscopic scale and so this cannot be excluded. (iii) In our experiments, the pieces cut from the originally circular mantle were flattened before the compression experiments were made. Possibly this distortion alters the resistance to compression. The distortion is greater for the larger pieces than for the smaller pieces.

\section{Relationship between work by muscle fibres and elastic energy}

It is of interest to compare the elastic energy that can be stored in the passive mantle with the amount of work done by the active circular muscle fibres as they contract and shorten. We can now make this comparison for Sepia officinalis, the only cephalopod for which both passive and active properties have been measured. However, the comparison is somewhat speculative because the experimental conditions were not identical in the two types of experiment.

Any work done in vivo to thicken the mantle would be provided by the active contraction of the circular muscle fibres. The amount of muscle fibre work that can be stored in this way can be evaluated from the relationship between strain and stress in the passive (non-contracting) mantle.

\section{Combining results for mantle pieces and intact mantle}

To obtain a complete picture of the relationship between stress and strain in the passive mantle, we need to combine the results of the two types of experiment reported here: results from compression of pieces of the mantle and pressure/volume results for the whole mantle. Both are required for the following reasons. (i) In the experiments involving compression of pieces of mantle, the measurements of stress were not sufficiently sensitive to detect the very small stresses that correspond to the flat, compliant region in the experiments on whole mantle (see Fig. 8). (ii) The large stresses reached in the compression experiment (see Fig. 5) could not be produced hydrostatically in the pressure/volume experiments.

There were two stages to combining the results shown in Figs 8 and 5. First the pressure/volume results were transformed to units of stress instead of pressure and strain instead of volume. The following assumptions were made. (i) Constant mantle length $(l)$, which was measured along the cuttlebone and was $115 \mathrm{~mm}$ for both Sepia officinalis used in the pressure/volume experiments; (ii) cylindrical shape for the mantle (see below for the effects of a rigid cuttlebone); and (iii) constant mantle wall volume $\left(V_{\mathrm{w}}\right)$. Wall volume was calculated from values measured when there was no pressure difference across the mantle (indicated by the subscript 0 ).

where

$$
V_{\mathrm{w}}=V_{\text {total }, 0}-V_{\text {cavity }, 0},
$$

$$
V_{\text {total }, 0}=l \pi\left(R_{\mathrm{i} 0}+t\right)^{2} .
$$

$R_{\mathrm{i} 0}$ is the radius of the cavity calculated from the volume of the cavity measured when there is no pressure difference across the mantle, and $t$ is the thickness of the mantle pieces, mean value $6.6 \mathrm{~mm}$, measured as described in the Materials and methods section. The mantle wall volumes for the two Sepia officinalis used in the pressure/volume experiments were 53788 and $57720 \mathrm{~mm}^{3}$.

Pressure was transformed to stress using the Laplace relationship:

$$
\sigma_{\text {wall }}=P R / t,
$$

where $\sigma_{\text {wall }}$ is wall stress, $P$ is observed pressure and $R$ is the average of the radius of the mantle cavity and the radius of the mantle to its outer surface, and $t$ is mantle wall thickness (see above).

For each pressure,

$$
R=0.5\left\{\sqrt{ }\left(V_{\mathrm{c}} / l \pi\right)+\sqrt{ }\left[\left(V_{\mathrm{c}}+V_{\mathrm{w}}\right) / l \pi\right]\right\},
$$

where $V_{\mathrm{c}}$ is the observed volume of the mantle cavity at pressure $P, V_{\mathrm{w}}$ is the mantle wall volume and $l$ is the length of the mantle.

Volumes were transformed to strain in the following way:

$$
\varepsilon=-l\left(c-c_{0}\right) / c_{0},
$$

where $\varepsilon$ is strain, $c$ is the average of the inner, $c_{\text {in }}$, and outer, $c_{\text {out }}$, circumferences of the mantle wall, and $c_{0}$ is the average circumference when there is zero pressure difference across the mantle wall.

$$
\begin{gathered}
c_{\text {in }}=2 \pi \sqrt{ }\left(V_{\mathrm{c}} / l \pi\right) \\
c_{\text {out }}=2 \pi \sqrt{ }\left[\left(V_{\mathrm{c}}+V_{\mathrm{w}}\right) / l \pi\right],
\end{gathered}
$$

where $V_{\mathrm{c}}$ is the observed volume of the mantle cavity at pressure $P, V_{\mathrm{w}}$ is the volume of the mantle wall and $l$ is the length of the mantle.

Second, the results for experiments on pieces of mantle have been shifted on the strain axis so that the stresses match those observed in the experiments on the whole mantle; the match was judged by eye. The combined results are shown in Fig. 9 in which the stress/strain relationship for compression in the circumferential direction (filled circles) has been shifted to the right along the strain axis. Similarly, the results for compression in the transverse direction (filled triangles) have been shifted to the left to match the stress.

\section{Effects of the rigid nature of the cuttlebone}

As indicated above, we have assumed that the mantle behaves like a cylinder of uniformly changeable circumference and constant length. However, the cuttlebone is rigid and prevents a section of the circumference from changing. To investigate the quantitative effects of the cuttlebone, we have 
calculated the stress/strain relationship assuming that part of the circumference of the cylinder is fixed and unable to change length. The results show that the strain in the remaining part of the mantle wall is greater at any given cavity volume than if we assume that the whole of the mantle wall participated in the change. The difference is apparent when the mantle enlarges beyond its neutral size (where there is zero pressure difference across the mantle wall), but there is very little difference when the mantle contracts beyond its neutral size (see Fig. 9A). Thus, the rigidity of the cuttlebone does not have much effect on the stress/strain relationship for positive strains, i.e. in the region where it has been proposed (Gosline and Shadwick, 1983a,b) that energy is stored elastically during the jet.

\section{Active and passive stress/strain relationships}

Fig. 10A shows how our stress/strain relationship for the passive, unstimulated tissue (filled circles) compares with the
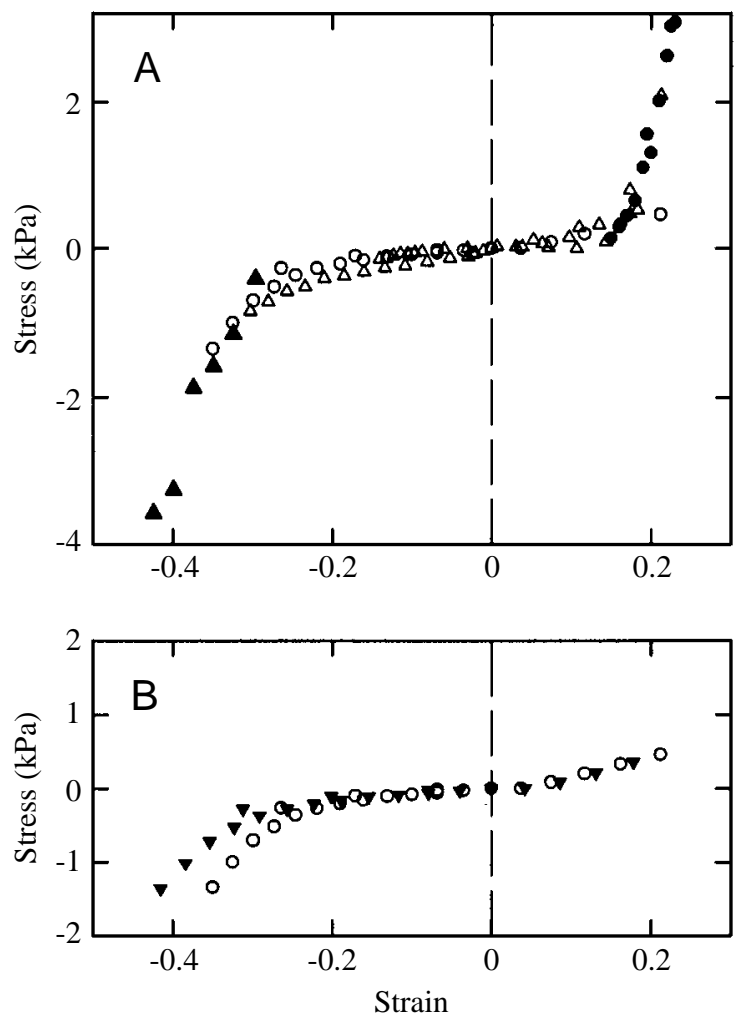

Fig. 9. (A) The relationship between stress and strain in experiments on whole mantle $(\triangle, \bigcirc$, see Fig. 8 and text). Also shown is the relationship for pieces of mantle compressed in the circumferential ( $)$ or in the transverse $(\boldsymbol{\Delta})$ direction. The points for the compression results have been positioned on the strain axis so that they match the relationship for the whole mantle. (B) The relationship between stress and strain in an experiment on whole mantle. Stress was calculated assuming either that the entire mantle circumference is elastic $(O$, as in $A)$ or assuming that part of the mantle circumference $(13 \mathrm{~mm})$ is the rigid cuttlebone and that the remaining part of the mantle circumference $(73 \mathrm{~mm}$ when not strained) is extensible and forms a circular $\operatorname{arc}(\boldsymbol{\nabla})$. See text for further details. stress/strain relationships of active slices of mantle wall (open circles). The relationship for passive tissue is that of which part is shown on an expanded scale in Fig. 9. The relationship for
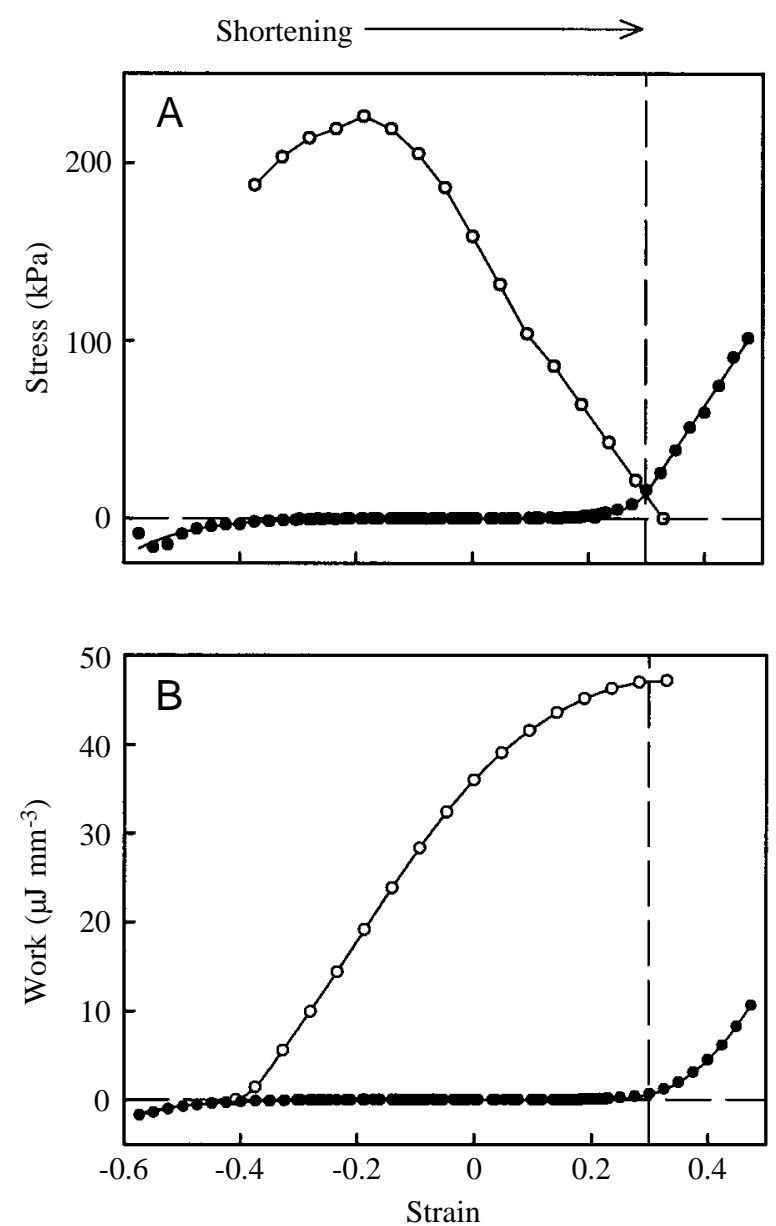

Fig. 10. The relationships between strain and stress (A) and work (B). Negative values of strain correspond to long circular muscle fibre lengths and positive values to short circular muscle fibre lengths. (A) Stress versus strain. Open symbols show the active stress produced by circular muscle fibres during stimulation under isometric (constant length) conditions (from Milligan et al., 1997). Filled symbols show the passive force exerted by the mantle (see Fig. 9 and text). The circular muscle fibres can shorten actively (from left to right) as far as the vertical broken line. Beyond this point, the passive resistance to shortening exceeds the stress that the muscle fibres can develop and no further shortening can occur. Strain zero corresponds to the length giving zero stress in Fig. 9 for the passive mantle and to the length giving zero passive force in the experiments of Milligan et al. (1997). (Note that Milligan et al. defined $L_{0}$ as the length giving maximum active force, and that a significant amount of passive force was produced at this length, as shown in their Fig. 5B. In the experiments of Milligan et al. (1977), passive force was zero at their $0.8 L_{0}$, as shown in their Fig. 5A.) (B) Work versus strain calculated from values shown in A. Open symbols show cumulative work done by active circular muscle fibres during shortening from a strain of -0.4 . Filled symbols show the cumulative elastic energy of the passive mantle during distortion from strain zero. Positive elastic work represents work done to decrease the circumference of the passive mantle. The vertical broken line shows the limit of active muscle shortening. 
active tissue is that reported by Milligan et al. (1997) for circular muscle fibres. The two sets of results were aligned so that the length giving zero stress in Fig. 9 for the passive mantle matches that giving zero passive force in the experiments of Milligan et al. (1997). Note that Milligan et al. (1997) defined $L_{0}$ as the length giving maximum active force (see their Fig. 5B); passive force was zero at $0.8 L_{0}$ (see their Fig. 5A).

Fig. 10B shows the work/strain relationships for passive mantle tissue and for active circular muscle fibres. The work done was calculated as the integral of stress with respect to strain. For the passive tissue, the values shown in Fig. 10A (filled symbols) were used. To calculate the work done by active muscle fibres, the stress was taken to be 0.4 of the isometric stresses shown by the open symbols in Fig. 10A. The value 0.4 is the ratio of the force produced during shortening to the isometric force, for shortening at $0.4 V_{\max }$, which is the velocity giving maximum power output in the squid Alloteuthis subulata (Milligan et al., 1997). The force/velocity relationship has not been investigated in Sepia officinalis.

The muscle fibres can shorten in vivo while the passive force resisting shortening is less than the active force developed by the muscle fibres. In Fig. 10A, the vertical broken line marks this limit; circular muscle fibres in the mantle cannot shorten into the region to the right of this line where passive force resisting shortening exceeds active force. The limit of circular muscle fibre shortening is also shown in Fig. 10B, where it intersects the work lines at the maximum values that can be reached. The maximum work that can be done by the circular muscle fibres during active shortening (open circles) is approximately $47.0 \mu \mathrm{J} \mathrm{mm}^{-3}$, and the maximum work that can be stored in the compressed tissue (filled circles) is approximately $0.5 \mu \mathrm{J} \mathrm{mm}^{-3}$. Thus, only approximately $1 \%$ of the work done by the muscle fibres can be stored elastically in the tissue.

An alternative method for aligning the two sets of results in Fig. 10A would be to match the resting stress in the muscle slices used by Milligan et al. (1997) to the passive mantle stress in Fig. 9. Milligan et al. (1977) found that the passive stress was $30 \mathrm{kPa}$ at the length giving maximum active stress. Thus, if this approach were used, the active muscle curves would be shifted by 0.43 strain units to the left of the position shown in Fig. 10A,B. In this case, the amount of work that could be stored elastically in the tissue would be considerably less than the $1 \%$ calculated on the basis of the alignment shown in Fig. 10A,B. However, the resting stress in the experiment of Milligan et al. (1977) was probably higher than in vivo because of the presence of a relatively high proportion of damaged muscle fibres in the small slices of mantle they used. For this reason, we think the second alignment method is less likely to represent the in vivo situation than the method used in Fig. 10A,B.

\section{Elastic energy storage and efficiency}

Gosline and Shadwick (1983b) and Shadwick (1994) concluded that a large fraction of the work done during muscle shortening could be stored in the compressed tissue. The conclusion was based on the assumption that the force remained constant while the muscle was actively producing force. This assumption is unlikely to be correct. Force recordings show that force changes continuously when the stimulation and movement patterns mimic those occurring during in vivo function. This has been found repeatedly in experiments on muscles from a wide variety of animals (e.g. Josephson, 1985; Stokes and Josephson, 1988; Curtin and Woledge, 1993a,b; Rome et al., 1993; Barclay, 1994; James and Johnston, 1998; Hammond et al., 1998). Nevertheless, we will examine further the hypothesis that elastic storage of work done during muscle shortening is functionally important.

The proposed function of energy storage in the compressed mantle is to provide elastic recoil to refill the mantle (Gosline and Shadwick, 1983b; Shadwick, 1994). Our evidence is that only a small amount of energy can be stored in the mantle. Is it sufficient to power refilling? In fact, the energy requirement for refilling is small; this is evident from the pressure and volume measurements during jetting of living squid, Loligo opalescens (see Fig. 3 in Gosline and Shadwick, 1983b) and L. forbesi (see Fig. 4 in Shadwick, 1994). Their observations show that refilling takes approximately the same time as ejection, but the pressure difference across the mantle during refilling is very much smaller than during ejection. The underlying functional anatomy is known: refilling requires a smaller pressure gradient because the water enters the mantle during refilling via the large lateral openings. Jetting requires a large pressure gradient because the water exits during jetting via the narrow siphon only (collar flaps close the lateral openings during jetting; Bone et al., 1994). Thus, the resistance to flow is much smaller during refilling than that during jetting. We have calculated the work done from the pressure and volume recordings reported by Gosline and Shadwick (1983b) and Shadwick (1994) and find that, in both cases, the work done during refilling is $1.3 \%$ of the work done during jetting. This is in good agreement with our value of $1.1 \%$ for the elastic work as a percentage of active muscle work (see above).

Gosline and Shadwick (1983b) and Shadwick (1994) proposed that the storage and use of elastic energy improve the energetic efficiency for the whole cycle of jetting and refilling. The efficiency of the jet cycle would not be improved by storing more energy than the very small amount required to refill the mantle $(1.3 \%$ of the work done during jetting in their experiments). All the stored elastic work represents work done by the circular muscle fibres, which necessarily incurs an energetic cost in the form of breakdown of ATP. This stored elastic energy can be used to do the work needed to refill the mantle, but any extra stored elastic energy in excess of the work required for refilling would be degraded into heat. Thus, the greatest efficiency would be achieved when the stored elastic work exactly matched the work required for refilling. Storing more elastic energy than needed would reduce energetic efficiency.

Finally, it should be noted that our conclusions are based largely on in vitro experimental results. We hope that other 


\section{N. A. Curtin, R. C. Woledge and Q. Bone}

investigators will be prompted to make further studies of living Sepia officinalis so that our conclusions can be tested using measurements of mantle stress and strain during in vivo function.

\section{References}

Barclay, C. J. (1994). Efficiency of fast- and slow-twitch muscle of the mouse performing cyclic contractions. J. Exp. Biol. 193, 65-73.

Bone, Q., Brown, E. R. and Travers, G. (1994). On the respiratory flow in the cuttlefish Sepia officinalis. J. Exp. Biol. 194, 153-165.

Bone, Q., Pulsford, A. and Chubb, A. D. (1981). Squid mantle muscle. J. Mar. Biol. Ass. UK 61, 327-342.

Curtin, N. A. and Woledge, R. C. (1993a). Efficiency of energy conversion during sinusoidal movment of white muscle fibres from the dogfish Scyliorhinus canicula. J. Exp. Biol. 183, 137-147.

Curtin, N. A. and Woledge, R. C. (1993b). Efficiency of energy conversion during sinusoidal movement of red muscle fibres from the dogfish Scyliorhinus canicula. J. Exp. Biol. 185, 195-206.

Gosline, J. M. and Shadwick, R. E. (1983a). Molluscan collagen and its mechanical organization in squid mantle. In Biochemistry of Mollusca (ed. P. W. Hochachka), pp. 371-398. New York: Academic Press.

Gosline, J. M. and Shadwick, R. E. (1983b). The role of elastic energy storage mechanisms in swimming: an analysis of mantle elasticity in escape jetting in the squid. Can. J. Zool. 61, 1421-1431.

Gosline, J. M., Steeves, J. D., Harman, A. D. and DeMont, M. E. (1983). Patterns of circular and radial mantle muscle activity in respiration and jetting of the squid Loligo opalescens. J. Exp. Biol. 104, 97-109.
Hammond, L., Altringham, J. D. and Wardle, C. S. (1998). Myotomal slow muscle function of rainbow trout Oncorhynchus mykiss during steady swimming. J. Exp. Biol. 201, 1659-1671.

James, R. S. and Johnston, I. A. (1998). Scaling of muscle performance during escape responses in the fish Myoxocephalus scorpius L. J. Exp. Biol. 201, 913-923.

Josephson, R. K. (1985). Mechanical power output from striated muscle during cyclic contraction. J. Exp. Biol. 114, 493-512.

Mendez, J. and Keys, A. (1960). Density and composition of mammalian muscle. Metabolism 9, 184-188.

Milligan, B. J., Curtin, N. A. and Bone, Q. (1997). Contractile properties of obliquely striated muscle from the mantle of squid (Alloteuthis subulata) and cuttlefish (Sepia officinalis). J. Exp. Biol. 200, 2425-2436.

Packard, A. and Trueman, E. R. (1974). Muscular activity of the mantle of Sepia and Loligo (Cephalopoda) during respiratory movements and jetting and its physiological interpretation. J. Exp. Biol. 61, 411-419.

Rome, L. C., Swank, D. and Corda, D. (1993). How fish power swimming. Science 261, 340-343.

Shadwick, R. E. (1994). Mechanical organization of the mantle and circulatory system of cephalopods. In Physiology of Cephalopod Molluscs (ed. H. O. Portner, R. O’Dor and D. L. Macmillan), pp. 69-85. Basel: Gordon \& Breach Publ.

Stokes, D. R. and Josephson, R. K. (1988). The mechanical power output of a crab respiratory muscle. J. Exp. Biol. 140, 287-299.

Ward, D. V. (1972). Locomotory function of the squid mantle. $J$. Zool., Lond. 167, 487-499.

Ward, D. V. and Wainwright, S. A. (1972). Locomotory aspects of squid mantle structure. J. Zool., Lond. 167, 437-449. 